Research Paper

\title{
The Efficacy and Safety of Anti-epidermal Growth Factor Receptor Monoclonal Antibodies in Nasopharyngeal Carcinoma: Literature-based Meta-analyses
}

\author{
Liang Peng ${ }^{*}$, Ze-Long Liu ${ }^{2 *}$, Cheng $\mathrm{Xu}^{1 *}$, Ling-Long Tang ${ }^{1}, \mathrm{Xu} \mathrm{Liu}^{1}$, Ai-Hua Lin ${ }^{3}$, Ying Sun ${ }^{1}$, Yu-Pei \\ Chen ${ }^{1 凶}$, Jun Ma ${ }^{\bowtie}$
}

1. Department of Radiation Oncology, Sun Yat-sen University Cancer Centre; State Key Laboratory of Oncology in South China; Collaborative Innovation Centre of Cancer Medicine; Guangdong Key Laboratory of Nasopharyngeal Carcinoma Diagnosis and Therapy, Guangzhou, China

2. Department of Medical Oncology, Sun Yat-sen University Cancer Centre; State Key Laboratory of Oncology in South China; Collaborative Innovation Centre of Cancer Medicine, Guangzhou, China

3. Department of Medical Statistics and Epidemiology, School of Public Health, Sun Yat-sen University, Guangzhou, China

* These authors contributed equally to this work.

$\square$ Corresponding authors: Jun Ma, Department of Radiation Oncology, Sun Yat-sen University Cancer Center, No. 651 Dongfeng Road East, Guangzhou 510060, China. Tel.:+86-20-87343469; Fax:+86-20-87343295; E-mail: majun2@mail.sysu.edu.cn and Yu-Pei Chen, Department of Radiation Oncology, Sun Yat-sen University Cancer Center, No. 651 Dongfeng Road East, Guangzhou 510060, China. Tel.:+86-20-87343469; Fax: +86-20-87343295; E-mail: chenyup1@sysucc.org.cn

(c) Ivyspring International Publisher. This is an open access article distributed under the terms of the Creative Commons Attribution (CC BY-NC) license (https://creativecommons.org/licenses/by-nc/4.0/). See http://ivyspring.com/terms for full terms and conditions.

Received: 2018.06.02; Accepted: 2018.09.10; Published: 2018.10.31

\begin{abstract}
Background: Anti-epidermal growth factor receptor monoclonal antibodies (anti-EGFR mAbs), such as cetuximab and nimotuzumab have been used in the treatment of nasopharyngeal carcinoma (NPC), yet their efficacy and safety are undetermined.

Materials and Methods: We performed two meta-analyses based on systematic searches of PubMed, EMBASE, the Cochrane Library and SinoMed: comparison 1 (standard therapy plus mAbs vs. standard therapy) and comparison 2 (radiotherapy plus concurrent mAbs vs. concurrent chemoradiotherapy) to explore the treatment value of anti-EGFR mAbs in NPC. Primary outcomes were overall survival (OS) and disease-free survival (DFS); secondary outcomes, locoregional recurrence-free survival (LRFS), distant metastasis-free survival (DMFS) and grade 3 and above acute adverse events.

Results: Four randomized controlled trials and thirteen observational studies were eligible. Comparison 1 (twelve studies): adding mAbs to standard therapy (radiotherapy or chemoradiotherapy) significantly improved OS (HR, 0.51; 95\% Cl, 0.39-0.66) and DFS (HR, 0.68; $95 \% \mathrm{Cl}, 0.54-0.86$ ), but increased the frequency of skin rashes and mucositis. Comparison 2 (six studies): $O S(\mathrm{HR}, 1.17 ; 95 \% \mathrm{Cl}, 0.81-1.70)$ and DFS $(\mathrm{HR}, 1.16 ; 95 \% \mathrm{Cl}, 0.86-1.57)$ were not significantly different when $\mathrm{mAbs}$ replaced conventional cytotoxic chemotherapy concurrently with radiotherapy, with fewer hematological, gastrointestinal and renal toxicities and more skin rashes in the mAb group.

Conclusion: We recommend anti-EGFR mAbs enhance-but should not replace-current treatment paradigms for locoregionally advanced NPC. Further evidence from phase III clinical trials is required.
\end{abstract}

Key words: nasopharyngeal carcinoma; anti-epidermal growth factor receptor; cetuximab; nimotuzumab; meta-analysis 


\section{Introduction}

Nasopharyngeal carcinoma (NPC) is very prevalent in endemic regions such as southern China, where the age-standardized annual incidence is 5-11 cases per 100,000 in endemic provinces, increasing to 10-27 cases in endemic counties [1]. In early-stage NPC (stage I), radiotherapy (RT) alone has good efficacy, and leads to a 5-year overall survival rate of over $90 \%$ [2]. However, up to $70 \%$ of patients present with locoregionally advanced (stage III-IV) disease at diagnosis [3]; RT combined with chemotherapy (chemoradiotherapy, CRT) is the recommended standard treatment for these patients [4, 5]. Yet, CRT fails in approximately $30 \%$ of patients; most treatment failures are due to distant metastasis [6, 7]. Moreover, conventional cytotoxic drugs (i.e., cisplatin, fluorouracil, taxol) are mainly selected for CRT, but can cause severe intolerable toxicities because of their unspecific cytotoxic effects in both normal and cancer cells [8].

Epidermal growth factor receptor (EGFR) is expressed in over $90 \%$ cases of NPC $[9,10]$, and a meta-analysis linked overexpression of EGFR to a poorer prognosis [11]. Activation of EGFR pathways promotes tumor cell growth, invasion and metastasis, prevents apoptosis and induces chemoresistance and radioresistance [12-15]. EGFR has been evaluated as a therapeutic target in NPC. Anti-EGFR monoclonal antibodies (anti-EGFR mAbs) bind to the extracellular domain of EGFR and prevent activation of downstream signaling pathways, and therefore exert anti-neoplastic effects. Of the several commercialized anti-EGFR mAbs available, cetuximab and nimotuzumab have been clinically tested in NPC. Several phase II single-arm clinical trials have preliminarily investigated the efficacy and toxicity of anti-EGFR mAbs in the treatment of NPC and reported promising results [16-19]. Moreover, one randomized controlled phase II trial demonstrated combining nimotuzumab and RT improved the complete response rate compared to RT alone in NPC, but no further survival outcomes were reported [20]. No data from phase III clinical trials is available to confirm the efficacy and toxicity of anti-EGFR mAbs in NPC; therefore, a comprehensive review of the available evidence is needed.

We conducted two separate head-to-head comparison meta-analyses in an attempt to answer the following questions: (1) whether combining anti-EGFR mAbs with the standard treatment (RT/CRT) improves efficacy without intolerable toxicities in NPC; and (2) whether anti-EGFR mAbs can replace conventional cytotoxic chemotherapy (usually platinum-based) in concurrent chemoradio- therapy (CCRT) with non-inferior efficacy and milder toxicities in NPC.

\section{Materials and Methods}

\section{Search strategy}

A comprehensive search was conducted of PubMed, EMBASE, the Cochrane Library and SinoMed (a Chinese database). We used the intelligent search functions provided by the electronic databases; the following terms and their synonyms provided by the intelligent search functions were used: "nasopharyngeal carcinoma" AND ("targeted therapy" OR "EGFR inhibitor" OR "anti-EGFR" OR "monoclonal antibody" OR "cetuximab" OR "nimotuzumab"). Abstracts from major cancer conferences were also considered in the EMBASE search. No restrictions on language or time of publication were applied. We also searched the US National Institutes of Health Trials Register and World Health Organization Clinical Trials Registry Platform for ongoing trials. The last search was performed on May 20th, 2018. We also manually searched the reference lists of retrieved papers or related reviews for additional studies.

\section{Outcomes}

We selected overall survival (OS) and disease-free survival (DFS) as primary outcomes and reported these as unadjusted hazard ratio (HR). Secondary outcomes were locoregional recurrencefree survival (LRFS) and distant metastasis-free survival (DMFS), reported as unadjusted HR, and grade 3 and above acute adverse events, reported as relative risk (RR). OS was defined as the time from treatment initiation to death from any cause; DFS, to distant metastasis, locoregional relapse or death from any cause (whichever first); LRFS, to locoregional relapse; DMFS, to distant metastasis. Acute adverse events were defined as occurring during treatment or within 90 days of treatment completion.

\section{Eligibility criteria}

Studies were included if they met all of the following predefined criteria: (1) patients with a pathological diagnosis of non-metastatic NPC without previous treatment; (2) head-to-head comparisons between RT/CRT plus anti-EGFR mAbs and RT/CRT alone, or RT plus concurrent anti-EGFR mAbs and RT plus concurrent chemotherapy; (3) studies reporting at least one of the primary outcomes (OS and DFS), as HR and associated 95\% confidence interval (CI) or other types of data (i.e., survival curves, numbers of events and log-rank $P$-values) from which we could calculate $\mathrm{HR}$ and $95 \% \mathrm{CI}$ indirectly. Reviews without original data, case 
reports, preclinical studies and single-arm studies were excluded. When cohorts from different studies overlapped, studies with the most participants or with the most complete data were included. The eligibility of each citation was independently assessed by two reviewers (L.P. and C.X.), and disagreements were resolved by discussion.

\section{Data extraction and quality assessment}

The following data was extracted and recorded using a predesigned table: first author, year of publication, study design, recruitment period, participants' age and sex, tumor stage and histologic type, sample size (per arm), treatment protocol, follow-up time, survival outcomes, and grade 3 and above acute adverse events. RCTs were assessed using the Cochrane risk of bias assessment tool and six domains for each included study were assessed: random sequence generation, allocation concealment, blinding (of participants and outcome assessors), outcome data, selective outcome reporting and other bias [21]. Studies were classified as having a low, unclear or high risk of bias. Observational studies were assessed with the modified Newcastle-Ottawa scale, which comprises three items: patient selection, comparability of the study groups, and assessment of outcomes [22]. Scores range from 0 to 9; studies with scores $\geq 6$ were regarded as high-quality. Two reviewers (L.P. and Z.L.L.) independently performed the extraction and assessment work and discussed any disagreements; a senior reviewer (Y.P.C.) was consulted if necessary.

\section{Statistical analysis}

For survival analysis, when unadjusted HR and associated $95 \%$ CI were unavailable, we used summary statistics or Kaplan-Meier curves to calculate the HR according to Parmar's method [23]. For adverse events analysis, the RR and $95 \%$ CI were used as outcome measures. Heterogeneity among studies was evaluated using the $\mathrm{Chi}^{2}$ test and $\mathrm{I}^{2}$ statistic. Heterogeneity was considered statistically significant if the $P$-value for the $\mathrm{Chi}^{2}$ test was $<0.10$ or $I^{2}$ value was $>50 \%$; if so, the random-effects model was used; otherwise, the fixed-effects model was used [24]. We entered HR data into the meta-analysis using the inverse variance method. Subgroup analyses were based on the mAb investigated, namely cetuximab or nimotuzumab. Funnel plots and Begg's tests were generated to detect potential publication bias using Stata software 12.0 (StataCorp, College Station, TX, USA), and $P>0.05$ was taken to indicate an absence of potential publication bias [25]. The meta-analysis and forest plots were produced using Review Manager 5.3 (Cochrane Collaboration, Oxford, UK).

\section{Results}

\section{Characteristics of studies}

Seventeen eligible studies were identified from the 1215 references retrieved [26-42]. The flow diagram of selection of the included studies is presented in Figure 1. Twelve studies [26-37] were included in the comparison between RT/CRT plus anti-EGFR mAbs and RT/CRT alone (comparison 1); six studies [29, 38-42] were included in the comparison between RT plus concurrent anti-EGFR mAbs and CCRT (comparison 2). Yin et al. [29] compared three cohorts: RT alone, RT plus concurrent anti-EGFR mAbs, and CCRT, so the comparison between RT alone and RT plus concurrent anti-EGFR $\mathrm{mAb}$ and the comparison between RT plus concurrent anti-EGFR $\mathrm{mAb}$ and CCRT were included in the respective meta-analyses. Among the studies included in comparison 1 , two were RCTs $[26,27]$ and ten were observational studies [28-37], and two studies [26, 29] used RT as standard treatment while the rest used CRT. You et al. [35] focusing on cetuximab/nimotuzumab and Xia et al. [34] focusing on cetuximab were conducted at the same institution and had partially overlapping participants, so we pooled the survival data from You et al. [35] in the overall analysis and Xia et al. [34] in the subgroup analysis. With regard to toxicities, data from You et al. [35] was pooled in the overall and subgroup analysis as adverse events were displayed individually for cetuximab/nimotuzumab. Among the studies included in comparison 2, two were RCTs $[38,39]$ and four were observational studies [29, 40-42]. The characteristics of the included studies are shown in Table 1 and the treatment protocols are summarized in Table 2.

\section{Assessment of included studies}

The results of the assessment are shown in Supplementary Tables S1 and S2. Wu et al. [26] reported single-center results from a multicenter, randomized controlled phase II trial [20], and was assessed as having an unclear risk of bias, as the two groups had a small, significant difference in age. The study by Shao et al. [27] was assessed as having a high risk of bias as the participants were allocated by drawing lots, which may cause non-neglectable selection bias. The phase II RCT registered as NCT01614938 by Xu et al. [38] was assessed as having an unclear risk, as it aimed to recruit at least 86 participants, but the trial was closed ahead of schedule because of the unexpectedly high rates of grade 3/4 mucositis observed in the RT plus concurrent cetuximab arm; this trial only recruited 44 participants. Liao et al. [39] reported the interim 
results of a phase III RCT registered as NCT02012062 and was assessed as having an unclear risk of bias. Of the thirteen observational studies, nine $[28,29,31,32$,
34-37, 40-42] were assessed as high-quality studies and two $[30,33]$ scored 5 indicating low quality.

Table 1. Characteristics of the included studies

\begin{tabular}{|c|c|c|c|c|c|c|c|c|c|c|}
\hline Study ID & $\begin{array}{l}\text { Study } \\
\text { type }\end{array}$ & $\begin{array}{l}\text { Recruitment } \\
\text { period }\end{array}$ & Study arms & $\begin{array}{l}\text { Sample } \\
\text { size }\end{array}$ & $\begin{array}{l}\text { Median } \\
\text { or mean } \\
\text { age } \\
\text { (years) }\end{array}$ & $\begin{array}{l}\text { Stage }(\%) / \text { staging } \\
\text { system }\end{array}$ & $\begin{array}{l}\text { Histologic } \\
\text { type } \\
\text { (WHO) }\end{array}$ & $\begin{array}{l}\text { Extractable } \\
\text { survival } \\
\text { outcomes }\end{array}$ & Toxicity criteria & $\begin{array}{l}\text { Median } \\
\text { follow-up } \\
\text { time } \\
\text { (months) }\end{array}$ \\
\hline \multicolumn{11}{|c|}{ Comparison 1: RT/CRT + anti-EGFR mAbs versus RT/CRT alone } \\
\hline Li 2015 & OB & 2009-2012 & $\begin{array}{l}\text { CCRT+NTZ } \\
\text { CCRT }\end{array}$ & $\begin{array}{l}30 \\
30\end{array}$ & $\begin{array}{l}57.3^{f} \\
54.6^{f}\end{array}$ & $\begin{array}{l}\text { II }(8.3 \%), \text { III }(38.3 \%), \\
\text { IV (53.4\%)/ Chinese } \\
2008 \text { staging }\end{array}$ & II, III & OS & CTCAEv4.0 & NR \\
\hline Lou 2016 & $\mathrm{OB}$ & 2012 & $\begin{array}{l}\mathrm{IC} \rightarrow \mathrm{CCRT}+\mathrm{NTZ} \\
\mathrm{IC} \rightarrow \mathrm{CCRT}\end{array}$ & $\begin{array}{l}22 \\
161\end{array}$ & NR & III (NR), IV (NR)/NR & NR & OS & $\begin{array}{l}\text { CTCAEv3.0, } \\
\text { RTOG/EORTC }\end{array}$ & 36 \\
\hline $\begin{array}{l}\text { Shao } \\
2014\end{array}$ & $\mathrm{RCT}$ & 2009-2010 & $\begin{array}{l}\text { CCRT+NTZ } \\
\text { CCRT }\end{array}$ & $\begin{array}{l}24 \\
24\end{array}$ & $\begin{array}{l}47 \mathrm{~g} \\
50 \mathrm{~g}\end{array}$ & 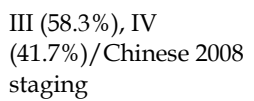 & II, III & OS,DFS & $\begin{array}{l}\text { CTCAEv } 4.0, \\
\text { RTOG }\end{array}$ & 26 \\
\hline $\begin{array}{l}\text { Tang } \\
2012\end{array}$ & OB & 2008-2011 & $\begin{array}{l}\mathrm{CCRT}+\mathrm{NTZ} \rightarrow \mathrm{AC} \\
\mathrm{CCRT} \rightarrow \mathrm{AC}\end{array}$ & $\begin{array}{l}63 \\
60\end{array}$ & $\begin{array}{l}54.2^{\mathrm{f}} \\
55.2^{\mathrm{f}}\end{array}$ & 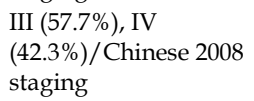 & NR & $\begin{array}{l}\text { OS,DFS,LRFS, } \\
\text { DMFS }\end{array}$ & $\begin{array}{l}\text { CTCAEv3.0, } \\
\text { RTOG/EORTC }\end{array}$ & 19.5 \\
\hline $\begin{array}{l}\text { Wang } \\
2016\end{array}$ & OB & 2008-2010 & $\begin{array}{l}\text { CCRT+CTX } \\
\text { CCRT }\end{array}$ & $\begin{array}{l}36 \\
42\end{array}$ & $\begin{array}{l}46.6^{f} \\
45.9^{f}\end{array}$ & $\begin{array}{l}\text { I-II }(80.8 \%), \text { III-IV } \\
(19.2 \%) / \mathrm{NR}\end{array}$ & NR & OS, DFS & RTOG & 54 \\
\hline $\begin{array}{l}\text { Wang } \\
2018\end{array}$ & OB & 2008-2014 & $\begin{array}{l}\mathrm{IC} \rightarrow \mathrm{CCRT}+\mathrm{NTZ} \rightarrow \\
\pm \mathrm{AC} \\
\mathrm{IC} \rightarrow \mathrm{CCRT} \rightarrow \pm \mathrm{AC}\end{array}$ & $\begin{array}{l}120 \\
120\end{array}$ & $\begin{array}{l}47 \mathrm{~g} \\
47 \mathrm{~g}\end{array}$ & $\begin{array}{l}\text { III }(59.6 \%), \text { IV } \\
(40.4 \%) / \text { AJCC } 7 \text { th } \\
\text { staging }\end{array}$ & NR & $\begin{array}{l}\text { OS,DFS,LRFS, } \\
\text { DMFS }\end{array}$ & $\begin{array}{l}\text { CTCAEv3.0, } \\
\text { RTOG }\end{array}$ & 56 \\
\hline Wu 2018 & $\mathrm{OB}$ & 2010-2014 & $\begin{array}{l}\text { CCRT+CTX } \\
\text { CCRT }\end{array}$ & $\begin{array}{l}75 \\
150\end{array}$ & $\begin{array}{l}47 \mathrm{~g} \\
47 \mathrm{~g}\end{array}$ & $\begin{array}{l}\text { III }(50.7 \%) \text {, IV } \\
(49.3 \%) / \text { AJCC } 7 \text { th } \\
\text { staging }\end{array}$ & II, III & $\begin{array}{l}\text { OS,DFS,LRFS, } \\
\text { DMFS }\end{array}$ & $\begin{array}{l}\text { CTCAEv3.0, } \\
\text { RTOG }\end{array}$ & $\begin{array}{l}41.0 \\
43.6\end{array}$ \\
\hline Wu 2007 & $\mathrm{RCT}$ & 2003-2004 & $\begin{array}{l}\mathrm{RT}+\mathrm{NTZ} \\
\mathrm{RT}\end{array}$ & $\begin{array}{l}18 \\
17\end{array}$ & $\begin{array}{l}36^{f} \\
47^{f}\end{array}$ & $\begin{array}{l}\text { III }(68.6 \%), \text { IV } \\
(31.4 \%) / \text { AJCC } 5 \text { th } \\
\text { staging }\end{array}$ & II, III & $\begin{array}{l}\text { OS,DFS,LRFS, } \\
\text { DMFS }\end{array}$ & $\begin{array}{l}\text { CTCAEv2.0, } \\
\text { RTOG }\end{array}$ & 31.9 \\
\hline Yin 2014a & $\mathrm{OB}$ & 2008-2012 & $\begin{array}{l}\text { RT+NTZ/CTX } \\
\text { RT }\end{array}$ & $\begin{array}{l}68 c \\
136\end{array}$ & NR & $\begin{array}{l}\text { II }(28.0 \%), \text { III }(38.2 \%) \text {, } \\
\text { IV }(33.8 \%) / \text { NR }\end{array}$ & NR & $\begin{array}{l}\text { OS,DFS,LRFS, } \\
\text { DMFS }\end{array}$ & CTCAEv3.0 & $31.3^{\mathrm{a}}$ \\
\hline You 2017 & OB & 2009-2013 & $\begin{array}{l}\text { CCRT+NTZ/CTX } \\
\text { CCRT }\end{array}$ & $\begin{array}{l}189 \mathrm{~d} \\
689\end{array}$ & $\begin{array}{l}44.7 \mathrm{~g} \\
45.6 \mathrm{~g}\end{array}$ & $\begin{array}{l}\text { II }(9.2 \%), \text { III }(71.4 \%) \\
\text { IV }(19.4 \%) / \text { AJCC } 7 \text { th } \\
\text { staging }\end{array}$ & II, III & $\begin{array}{l}\text { OS,DFS,LRFS, } \\
\text { DMFS }\end{array}$ & $\begin{array}{l}\text { CTCAEv4.0, } \\
\text { RTOG/EORTC }\end{array}$ & $\begin{array}{l}48.0 \\
48.9\end{array}$ \\
\hline $\begin{array}{l}\text { Zeng } \\
2016\end{array}$ & $\mathrm{OB}$ & 2010-2012 & $\begin{array}{l}\text { CCRT+CTX } \\
\text { CCRT }\end{array}$ & $\begin{array}{l}64 \\
74\end{array}$ & $\begin{array}{l}45.3^{f} \\
45.0^{f}\end{array}$ & $\begin{array}{l}\text { III }(47.8 \%), \text { IV } \\
(52.2 \%) / \text { AJCC } 6 \text { th } \\
\text { staging }\end{array}$ & NR & OS & NR & NR \\
\hline Xia 2017b & OB & 2006-2013 & $\begin{array}{l} \pm \mathrm{IC} \rightarrow \mathrm{CCRT}+\mathrm{CTX} \\
\pm \mathrm{IC} \rightarrow \mathrm{CCRT}\end{array}$ & $\begin{array}{l}96 \\
96\end{array}$ & $\begin{array}{l}43.9^{f} \\
44.0^{f}\end{array}$ & $\begin{array}{l}\text { III (NR), IV } \\
\text { (NR)/ AJCC 7th } \\
\text { staging }\end{array}$ & II, III & $\begin{array}{l}\text { OS,DFS,LRFS, } \\
\text { DMFS }\end{array}$ & CTCAEv4.0 & $\begin{array}{l}62 \\
62.9\end{array}$ \\
\hline \multicolumn{11}{|c|}{ Comparison 2: RT + concurrent anti-EGFR mAbs versus CCRT } \\
\hline Li 2016 & OB & 2008-2013 & $\begin{array}{l}\mathrm{IC} \rightarrow \mathrm{RT}+\mathrm{NTZ} \\
\mathrm{IC} \rightarrow \mathrm{CCRT}\end{array}$ & $\begin{array}{l}52 \\
52\end{array}$ & NR & $\begin{array}{l}\text { II }(25.0 \%), \text { III }(39.4 \%) \text {, } \\
\text { IV }(35.6 \%) / \text { AJCC } 7 \text { th } \\
\text { staging }\end{array}$ & II, III & OS,DFS & CTCAEv4.0 & 50 \\
\hline Liao 2016 & $\mathrm{RCT}$ & 2012-2013 & $\begin{array}{l}\mathrm{IC} \rightarrow \mathrm{RT}+\mathrm{NTZ} \\
\mathrm{IC} \rightarrow \mathrm{CCRT}\end{array}$ & $\begin{array}{l}28 \\
32\end{array}$ & $47 \mathrm{~g}$ & $\begin{array}{l}\text { III }(51.7 \%), \text { IV } \\
(48.3 \%) / \text { AJCC } 7 \text { th } \\
\text { staging }\end{array}$ & II, III & $\begin{array}{l}\text { OS,DFS,LRFS, } \\
\text { DMFS }\end{array}$ & CTCAEv3.0 & 36 \\
\hline Wu 2016 & $\mathrm{OB}$ & 2008-2012 & $\begin{array}{l}\mathrm{IC} \rightarrow \mathrm{RT}+\mathrm{CTX} \\
\mathrm{IC} \rightarrow \mathrm{CCRT}\end{array}$ & $\begin{array}{l}56 \\
56\end{array}$ & $\begin{array}{l}46.2 \mathrm{~g} \\
45.8 \mathrm{~g}\end{array}$ & $\begin{array}{l}\text { II }(17.0 \%), \text { III }(49.1 \%) \\
\text { IV }(33.9 \%) / \text { AJCC } 7 \text { th } \\
\text { staging }\end{array}$ & II, III & OS,DFS & CTCAEv4.0 & $\begin{array}{l}55.4 \\
56.2\end{array}$ \\
\hline Xu 2015 & RCT & 2010-2011 & $\begin{array}{l}\mathrm{IC} \rightarrow \mathrm{RT}+\mathrm{CTX} \\
\mathrm{IC} \rightarrow \mathrm{CCRT}\end{array}$ & $\begin{array}{l}21 \\
23\end{array}$ & $\begin{array}{l}44 g \\
46 g\end{array}$ & $\begin{array}{l}\text { III }(70.5 \%), \text { IV } \\
(29.5 \%) / \text { AJCC } 7 \text { th } \\
\text { staging }\end{array}$ & II, III & $\begin{array}{l}\text { OS,DFS,LRFS, } \\
\text { DMFS }\end{array}$ & CTCAEv3.0 & 36.9 \\
\hline Yin 2014a & OB & 2008-2012 & $\begin{array}{l}\mathrm{RT}+\mathrm{NTZ} / \mathrm{CTX} \\
\text { CCRT }\end{array}$ & $\begin{array}{l}68 c \\
136\end{array}$ & NR & $\begin{array}{l}\text { II }(28.0 \%), \text { III }(38.2 \%) \text {, } \\
\text { IV }(33.8 \%) / \text { NR }\end{array}$ & NR & $\begin{array}{l}\text { OS,DFS,LRFS, } \\
\text { DMFS }\end{array}$ & CTCAEv3.0 & $31.3^{a}$ \\
\hline $\begin{array}{l}\text { You-Rui } \\
2017\end{array}$ & $\mathrm{OB}$ & 2009-2013 & $\begin{array}{l} \pm \mathrm{IC} \rightarrow \mathrm{RT}+\mathrm{NTZ} / \mathrm{CT} \\
\mathrm{X} \\
\pm \mathrm{IC} \rightarrow \mathrm{CCRT}\end{array}$ & $\begin{array}{l}143 e \\
572\end{array}$ & $\begin{array}{l}46 g \\
47 g\end{array}$ & $\begin{array}{l}\text { II }(11.2 \%), \text { III }(55.7 \%) \text {, } \\
\text { IV }(33.9 \%) / \text { AJCC } 7 \text { th } \\
\text { staging }\end{array}$ & II, III & $\begin{array}{l}\text { OS,DFS,LRFS, } \\
\text { DMFS }\end{array}$ & $\begin{array}{l}\text { CTCAEv4.0, } \\
\text { RTOG }\end{array}$ & $\begin{array}{l}50.4 \\
55.1\end{array}$ \\
\hline $\begin{array}{l}\text { Abbreviatio } \\
\text { CRT = chem } \\
\text { NR = not re } \\
\text { distant meta } \\
\text { Organizatio } \\
\text { total three c } \\
\text { DFS. c Numb } \\
\text { NTZ/CTX }\end{array}$ & $\begin{array}{l}\text { ons: OB } \\
\text { moradiot } \\
\text { eported; } \\
\text { tastasis-f } \\
\text { on for Re } \\
\text { cohorts. } \\
\text { abers of }\end{array}$ & $\begin{array}{l}=\text { observatiol } \\
\text { therapy; } \mathrm{CCF}\end{array}$ & $\begin{array}{l}\text { l; RCT }=\text { randomized c } \\
=\text { concurrent chemora } \\
\text { ican Joint Committee o } \\
\text { TCAE = Common Ter } \\
\text { atment of Cancer. }{ }^{\text {a Yin }}\end{array}$ & $\begin{array}{l}\text { ontrolled } \\
\text { diotherap } \\
\text { n Cancer; } \\
\text { minology } \\
2014 \text { with }\end{array}$ & $\begin{array}{l}\text { anti-EGFI } \\
=\text { inducti } \\
\text { overall st } \\
\text { ria for Ad } \\
\text { e cohorts } \\
\text { ed with } \mathrm{tl}\end{array}$ & $\begin{array}{l}\text { mAbs = anti-epidermal } \\
\text { on chemotherapy; } \mathrm{AC}=\mathrm{a} \\
\text { arvival; DFS = disease-fre } \\
\text { verse Events; RTOG = Ra } \\
\text { was split into two compar } \\
\text { he cohort of You 2017, and } \\
\text { articipants treated with N }\end{array}$ & isons, and the & $\begin{array}{l}\text { therapy; } N Z=1 \\
S=\text { locoregional } \\
\text { y Oncology Grou } \\
\text { median follow-up } \\
\text { uded in cetuxima } \\
37 / 102 . \text { e Number }\end{array}$ & $\begin{array}{l}\text { nimotuzumab; CT } \\
\text { recurrence-free sur } \\
\text { p; EORTC = Europ } \\
\text { o time was calculat } \\
\text { b subgroup analys } \\
\text { is of participants tr }\end{array}$ & $\begin{array}{l}\text { radiotherapy; } \\
=\text { cetuximab; } \\
\text { ival; DMFS = } \\
\text { an } \\
\text { based on the } \\
\text { s for OS and } \\
\text { ted with }\end{array}$ \\
\hline
\end{tabular}


Table 2. Treatment protocols of the included studies

\begin{tabular}{|c|c|c|c|}
\hline Study ID & Radiotherapy & Chemotherapy & Anti-EGFR mAb during RT \\
\hline \multicolumn{4}{|c|}{ Comparison 1: RT/CRT + anti-EGFR mAbs versus RT/CRT alone } \\
\hline Li 2015 & 3DCRT:60-70Gy & CCRT: NDP 40 mg qw for 6 cycles & NTZ $200 \mathrm{mg}$ qw for 6 cycles \\
\hline Lou 2016 & IMRT:66-70Gy & $\begin{array}{l}\text { IC: TP or TPF q3w for } 1-2 \text { cycles; } \\
\text { CCRT: NDP } 80 \mathrm{mg} / \mathrm{m}^{2} \mathrm{q} 3 \mathrm{w} \text { for } 2 \text { cycles }\end{array}$ & NTZ $100 \mathrm{mg}$ or $200 \mathrm{mg}$ qw for 6 cycles \\
\hline Shao 2014 & 3DCRT:72-76Gy & CCRT: NDP $30 \mathrm{mg} / \mathrm{m}^{2} \mathrm{qw}$ for 7 cycles & NTZ 100 mg qw for 7 cycles \\
\hline Tang 2012 & $\begin{array}{l}\text { 3DCRT:70-76Gy } \\
\text { IMRT:70-76Gy }\end{array}$ & $\begin{array}{l}\text { CCRT: TP q3w for } 2-3 \text { cycles; } \\
\text { AC: TP q3w for } 2-4 \text { cycles }\end{array}$ & NTZ $100 \mathrm{mg}$ qw for 6-7 cycles \\
\hline Wu 2007 & 2DRT:70Gy & NA & NTZ 100 mg qw for 8 cycles \\
\hline Wang 2016 & IMRT:66Gy & CCRT: CDDP $40 \mathrm{mg} / \mathrm{m}^{2} \mathrm{qw}$ for 6 cycles & CTX $40 \mathrm{mg} / \mathrm{m}^{2} \mathrm{qw}$ for 6 cycles \\
\hline Wang 2018 & IMRT:66-72Gy & $\begin{array}{l}\text { IC: TP or PF or TPF or GP q3w for } 2-3 \text { cycles; } \\
\text { CCRT: CDDP } 80 \mathrm{mg} / \mathrm{m}^{2} \mathrm{q} 3 \mathrm{w} \text { for } 2-3 \text { cycles; } \\
\text { AC: PF or GP }\end{array}$ & NTZ 200 mg qw for 6-7 cycles \\
\hline Wu 2018 & IMRT:66-75Gy & $\begin{array}{l}\text { CCRT: TP or CDDP } 80 \mathrm{mg} / \mathrm{m}^{2} \text { or NDP } 80 \mathrm{mg} / \mathrm{m}^{2} \mathrm{q} 3 \mathrm{w} \\
\text { for } 2 \text { cycles }\end{array}$ & CTX $400 \mathrm{mg}$ loading, $250 \mathrm{mg}$ qw for 7 cycles \\
\hline Yin 2014 & IMRT:70-74Gy & NA & $\begin{array}{l}\text { CTX } 400 \mathrm{mg} \text { loading, } 250 \mathrm{mg} \text { qw for } 7 \text { cycles; } \\
\text { NTZ } 200 \mathrm{mg} \text { qw for } 8 \text { cycles }\end{array}$ \\
\hline You 2017 & IMRT:68-72Gy & CCRT: CDDP $80-100 \mathrm{mg} / \mathrm{m}^{2} \mathrm{q} 3 \mathrm{w}$ for $2-3$ cycles & $\begin{array}{l}\text { CTX } 400 \mathrm{mg} \text { loading, } 250 \mathrm{mg} \text { qw for 6-7 cycles; } \\
\text { NTZ } 200 \mathrm{mg} \text { qw for 6-7 cycles }\end{array}$ \\
\hline Zeng 2016 & IMRT:70Gy & CCRT: CDDP $40 \mathrm{mg} / \mathrm{m}^{2} \mathrm{qw}$ for 6 cycles & CTX $400 \mathrm{mg}$ loading, $250 \mathrm{mg}$ qw for 7 cycles \\
\hline Xia 2017 & $\begin{array}{l}\text { 2DRT:70-76Gy } \\
\text { IMRT:68-72Gy }\end{array}$ & $\begin{array}{l}\text { CCRT: CDDP } 80-100 \mathrm{mg} / \mathrm{m}^{2} \mathrm{q} 3 \mathrm{w} \text { for } 2-3 \text { cycles or } \\
30-40 \mathrm{mg} / \mathrm{m}^{2} \mathrm{qw} \text { for } 5-7 \text { cycles }\end{array}$ & CTX $400 \mathrm{mg}$ loading, $250 \mathrm{mg}$ qw for 6-7 cycles \\
\hline \multicolumn{4}{|c|}{ Comparison 2: RT + concurrent anti-EGFR mAbs versus CCRT } \\
\hline Li 2016 & IMRT:70-74Gy & $\begin{array}{l}\text { IC: TPF q3w for } 2 \text { cycles; } \\
\text { CCRT: CDDP } 75 \mathrm{mg} / \mathrm{m}^{2} \mathrm{q} 3 \mathrm{w} \text { for } 2-3 \text { cycles }\end{array}$ & NTZ $200 \mathrm{mg}$ qw for $6-8$ cycles \\
\hline Liao 2016 & IMRT:70Gy & $\begin{array}{l}\text { IC: TPF q3w for } 3 \text { cycles; } \\
\text { CCRT: CDDP } 40 \mathrm{mg} / \mathrm{m}^{2} \mathrm{qw} \text { for } 7 \text { cycles }\end{array}$ & NTZ $200 \mathrm{mg}$ qw for 8 cycles \\
\hline Wu 2016 & IMRT:70-74Gy & $\begin{array}{l}\text { IC: TPF q3w for } 2 \text { cycles; } \\
\text { CCRT: CDDP } 75 \mathrm{mg} / \mathrm{m}^{2} \mathrm{q} 3 \mathrm{w} \text { for } 2-3 \text { cycles }\end{array}$ & CTX $400 \mathrm{mg}$ loading, $250 \mathrm{mg}$ qw for 8 cycles \\
\hline Xu 2015 & IMRT:66-70Gy & $\begin{array}{l}\text { IC: TP q3w for } 2 \text { cycles; } \\
\text { CCRT: CDDP } 30 \mathrm{mg} / \mathrm{m}^{2} \mathrm{qw}\end{array}$ & CTX $250 \mathrm{mg}$ qw for 7 cycles \\
\hline Yin 2014 & IMRT:70-74Gy & CCRT: CDDP qw for 6 cycles or q3w for 2 cycles & $\begin{array}{l}\text { CTX } 400 \text { mg loading, } 250 \text { mg qw for } 7 \text { cycles; } \\
\text { NTZ } 200 \mathrm{mg} \text { qw for } 8 \text { cycles }\end{array}$ \\
\hline You-Rui 2017 & IMRT:76-70Gy & $\begin{array}{l}\text { IC: TP or PF or TPF q3w for } 2-3 \text { cycles; } \\
\text { CCRT: CDDP } 80-100 \mathrm{mg} / \mathrm{m}^{2} \mathrm{q} 3 \mathrm{w} \text { for } 2-3 \text { cycles }\end{array}$ & $\begin{array}{l}\text { CTX } 400 \mathrm{mg} \text { loading, } 250 \mathrm{mg} \text { qw for } 6-7 \text { cycles; } \\
\text { NTZ } 200 \mathrm{mg} \text { qw for } 6-7 \text { cycles }\end{array}$ \\
\hline
\end{tabular}

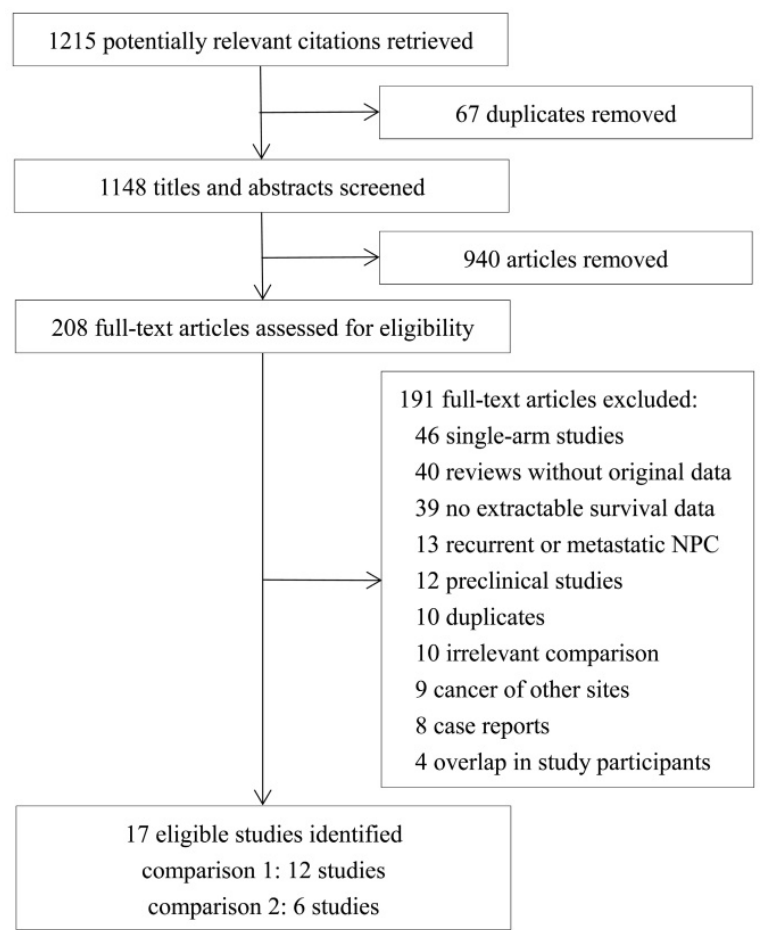

Figure 1. Flow diagram of selection of included and excluded studies.

\section{Comparison 1: RT/CRT plus anti-EGFR mAbs vs. RT/CRT alone}

Pooled HR for OS showed evidence of a benefit of adding anti-EGFR mAbs to standard therapy (HR, 0.51; 95\% CI, 0.39-0.66). Pooled analysis for DFS showed a benefit favoring addition of anti-EGFR mAbs (HR, 0.68; 95\% CI, 0.54-0.86). Pooled analysis for LRFS also indicated a benefit favoring addition of anti-EGFR mAbs (HR, 0.66; 95\% CI, 0.45-0.96). With regards to DMFS, a marginally significant effect was observed (HR, 0.64; 95\% CI, 0.38-1.07). No significant heterogeneity was detected, except in the analysis of DMFS $\left(I^{2}=53 \%, P=0.06\right.$; Figure 2$)$.

Ten types of adverse event were evaluated (Table 3). Grade 3 and above skin rashes and mucositis were more frequently observed in participants treated with RT/CRT plus anti-EGFR mAbs. The pooled RR $(95 \% \mathrm{CI})$ for skin rashes and mucositis were 4.08 (1.59-10.47) and 1.84 (1.10-3.10) respectively; both had significant heterogeneity.

In the cetuximab subgroup, OS and DFS remained significantly improved by adding cetuximab to 
standard therapy compared to standard therapy alone; pooled RR $(95 \% \mathrm{CI})$ for skin rashes and mucositis were 7.28 (2.17-24.43) and 2.31 (1.04-5.15). In the nimotuzumab subgroup, OS was significantly improved by adding nimotuzumab, though the improvement in DFS was marginally significant (HR, 0.65; 95\% CI, 0.41-1.03; $P=0.06)$; pooled RR (95\% CI) for skin rashes and mucositis were 1.19 (0.51-2.78) and 1.25 (0.81-1.94; Supplementary Table S3).

\section{Comparison 2: RT plus concurrent anti-EGFR mAbs vs. CCRT}

Pooled analysis showed the survival differences between the two modalities were not significant (OS: HR, 1.17; 95\% CI, 0.81-1.70; DFS: HR, 1.16; 95\% CI,
0.86-1.57; LRFS: HR, 0.83; 95\% CI, 0.44-1.58; DMFS: $\mathrm{HR}, 1.17$; $95 \% \mathrm{CI}, 0.73-1.85)$ and no significant heterogeneity was detected (Figure 3).

Ten types of adverse events were evaluated (Table 3). Compared to CCRT, RT plus concurrent anti-EGFR mAbs was less likely to induce grade 3 and above leucopenia (RR, 0.23; 95\% CI, 0.10-0.53), thrombocytopenia (RR, 0.27; 95\% CI, 0.11-0.62), anemia (RR, 0.09; 95\% CI, 0.03-0.33), nausea/vomiting (RR, 0.10; 95\% CI, 0.05-0.18) and renal function abnormality (RR, 0.20; 95\% CI, 0.05-0.79), but more likely to cause grade 3 and above skin rashes (RR, 4.09 ; $95 \%$ CI, 1.21-13.87).

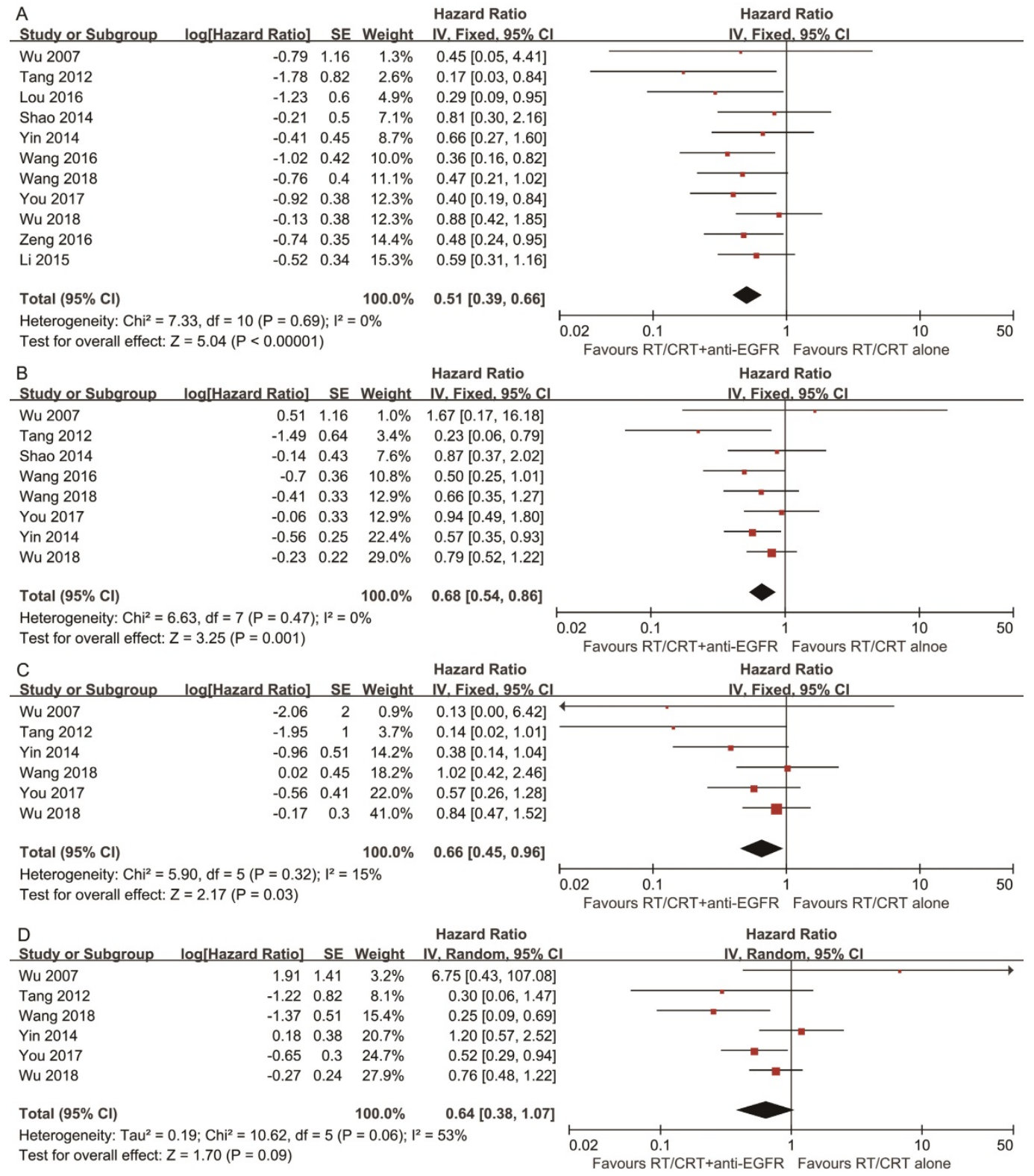

Figure 2. Forest plots of the overall analyses for comparison 1, comparing RT/CRT plus anti-EGFR mAbs and RT/CRT alone. (A) Overall survival; (B) disease-free survival; (C) locoregional recurrence-free survival; (D) distant metastasis-free survival. $\mathrm{RT}=$ radiotherapy; CRT = chemoradiotherapy; anti-EGFR mAbs = anti-epidermal growth factor receptor monoclonal antibodies. 


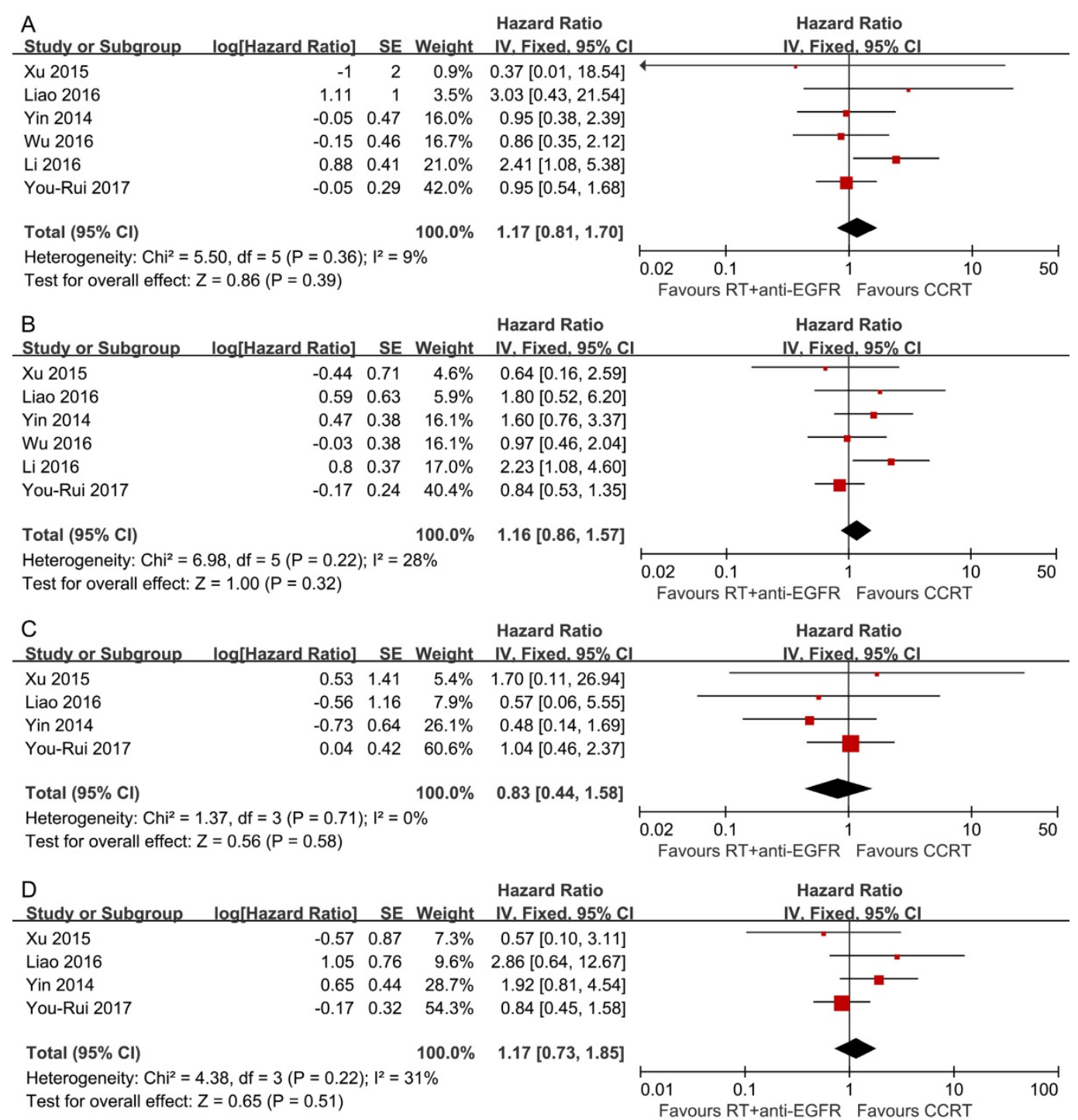

Figure 3. Forest plots of the overall analyses for comparison 2, comparing RT plus concurrent anti-EGFR mAbs and CCRT. (A) Overall survival; (B) disease-free survival; (C) locoregional recurrence-free survival; (D) distant metastasis-free survival. $R T$ = radiotherapy; CCRT = concurrent chemoradiotherapy; anti-EGFR mAbs $=$ anti-epidermal growth factor receptor monoclonal antibodies.

In the cetuximab subgroup, OS and DFS remained non-significantly different between RT plus concurrent cetuximab and CCRT; pooled RR (95\% CI) for skin rashes and mucositis were 11.13 (6.16-20.10) and 1.62 (1.33-1.98). In the nimotuzumab subgroup, pooled HR (95\% CI) for OS and DFS were 2.49 (1.18-5.24) and 2.11 (1.13-3.94), indicating concurrent nimotuzumab has inferior efficacy compared to CCRT; pooled RR $(95 \% \mathrm{CI})$ for skin rashes and mucositis were $1.32(0.22-8.06)$ and $0.92(0.72-1.18$; Supplementary Table S4).

In both comparisons 1 and 2, the results of the sensitivity analyses for primary outcomes (OS and DFS) were in accordance with the overall analyses, suggesting the results were robust (Supplementary Tables S5 and S6). Additionally, no significant publication bias was detected in the overall analyses for OS and DFS (Figure 4).

\section{Discussion}

\section{Comparison 1: anti-EGFR mAbs can enhance the efficacy when combined to standard therapy in the treatment of locoregionally advanced NPC}

According to the results of our meta-analyses, addition of anti-EGFR mAbs to standard therapy for NPC improved efficacy in terms of OS, DFS and LRFS compared to standard therapy alone, and sensitivity analyses confirmed the robustness of these results. This result can be explained by the synergetic anti-neoplastic effects of anti-EGFR mAbs combined with chemotherapy and radiotherapy, which renders NPC cells more sensitive to cytotoxic drugs and ionizing radiation $[43,44]$. However, the improvement in DMFS was insignificant, which may be due to 
the between-study heterogeneity of the four studies included. When interpreting improvements in efficacy, caution is necessary due to the following constraints of the included studies: (1) most participants had advanced stage NPC (stage III and IV accounted for $90.7 \%$ ); (2) the median follow-up time for the included studies ranged from 19.5 to 56 months, and only five studies [31, 32, 35-37] had a median follow-up time $\geq 36$ months; (3) most studies were conducted using CRT as standard therapy, and only two studies [26, 29] employed RT. At present, there is still a lack of evidence to prove the superiority of combining anti-EGFR mAbs with RT over RT alone in stage III and IV disease. Indeed, CRT is recommended as the standard therapy for stage III and IV NPC and RT alone is sub-optimal, and only advisable if chemotherapy cannot be administered due to contraindications. For patients with stage II NPC, disagreements still exist regarding the optimal treatment modality (RT or CRT) due to the intrinsic heterogeneity of this group of patients [45]. Therefore, whether it is suitable to add anti-EGFR mAbs to RT/CRT in stage II NPC or not is a question that needs to be addressed in the future. On the other hand, RT plus anti-EGFR mAbs could be an
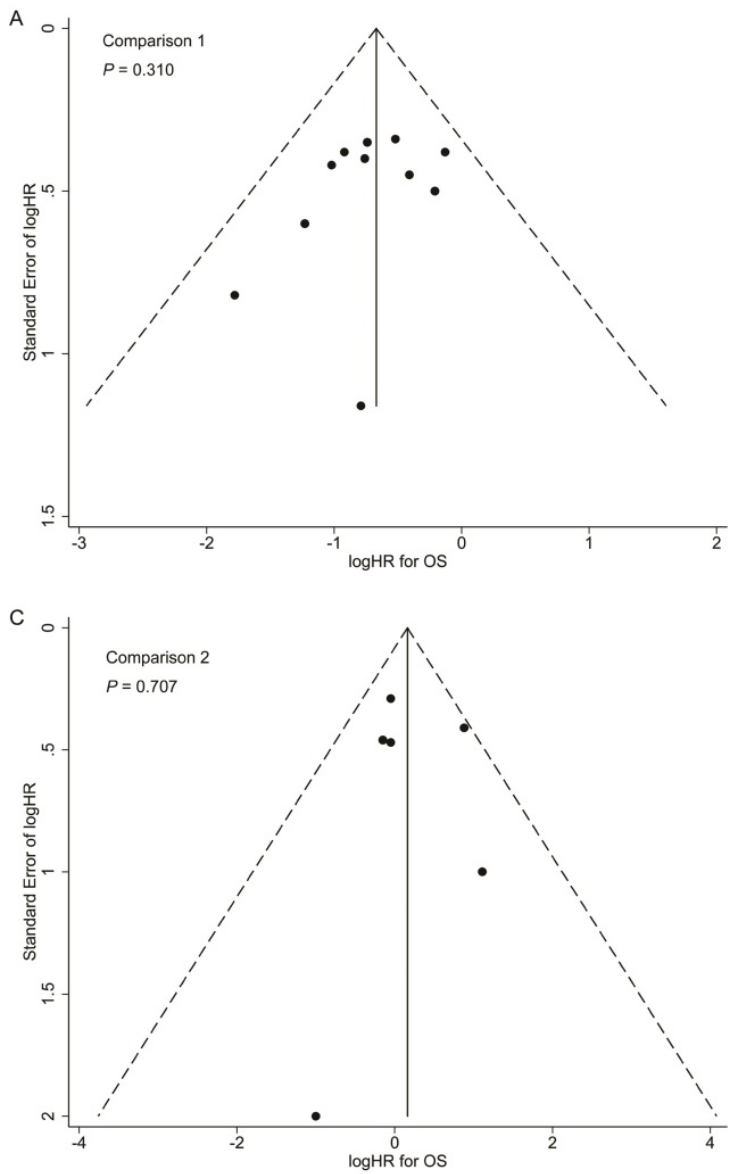

alternative to RT or CRT for stage II NPC (see below). When interpreting the results of subgroup analyses for survival outcomes, we should bear in mind that some studies investigating both cetuximab and nimotuzumab were not included in the subgroup analyses as detailed data was not provided separately for each $\mathrm{mAb}$, and this may explain why the improvement in DFS in the nimotuzumab subgroup was not as significant as in the overall analyses.

Adding anti-EGFR mAbs to standard therapy was more likely to cause grade 3 and above skin rashes and mucositis compared with standard therapy alone. This can be explained by the fact that EGFR mAbs can bind to some normal cells, such as the epidermis and mucosal epithelium [46]. However, such adverse events resolved after withdrawal of the mAbs due to the self-repairing ability of epithelial tissue [47]. To some extent, subgroup analyses revealed nimotuzumab was less likely to cause such mAb-related adverse events, which can be explained by the lower EGFR-binding affinity of nimotuzumab compared to cetuximab [48], though this needs to be confirmed by direct or indirect comparisons between nimotuzumab and cetuximab.
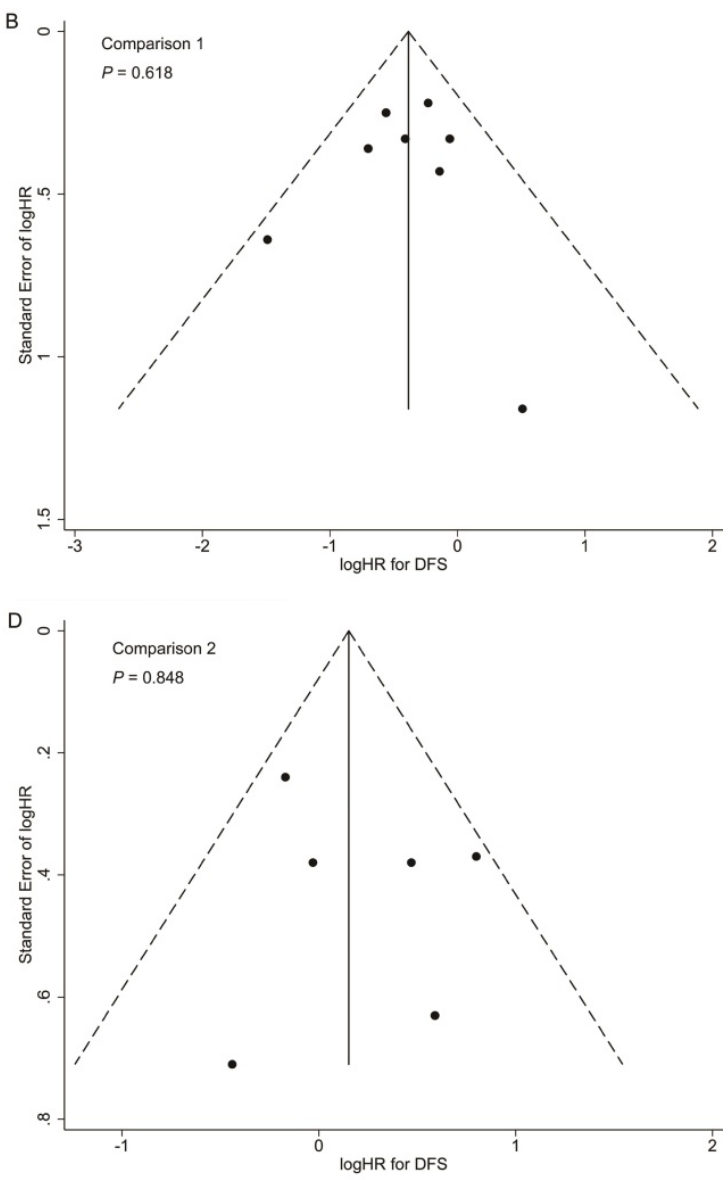

Figure 4. Funnel plots with pseudo $95 \%$ confidence limits of overall analyses for comparison 1 (A: overall survival; B: disease-free survival) and comparison 2 (C: overall survival; D: disease-free survival). $P$-values were calculated by Begg's test. 
Table 3. Meta-analyses of grade 3 and above adverse events for comparisons 1 and 2

\begin{tabular}{|c|c|c|c|c|c|c|c|}
\hline \multirow[t]{2}{*}{ Adverse events } & \multirow[t]{2}{*}{$\mathrm{N}$ of studies } & \multirow{2}{*}{$\begin{array}{l}\mathrm{N} \text { of pts in } \mathrm{mAb} \\
\text { group }\end{array}$} & \multirow{2}{*}{$\begin{array}{l}\mathrm{N} \text { of pts in control } \\
\text { group }\end{array}$} & \multirow[t]{2}{*}{ RR $(95 \%$ CI $)$} & \multirow[t]{2}{*}{$P$-value ${ }^{a}$} & \multicolumn{2}{|c|}{ Heterogeneity } \\
\hline & & & & & & $I^{2}$ & $P$-value \\
\hline \multicolumn{8}{|c|}{ Comparison 1: RT/CRT + anti-EGFR mAbs versus RT/CRT alone } \\
\hline Leukopenia & 7 & 565 & 1147 & $0.99(0.83-1.19)$ & 0.95 & $11 \%$ & 0.34 \\
\hline Thrombocytopenia & 5 & 438 & 1013 & $1.01(0.53-1.95)$ & 0.97 & $13 \%$ & 0.33 \\
\hline Anemia & 5 & 477 & 1049 & $1.21(0.68-2.15)$ & 0.52 & $9 \%$ & 0.35 \\
\hline Nausea/vomiting & 7 & 565 & 1147 & $0.95(0.58-1.56)$ & 0.84 & $46 \%$ & 0.09 \\
\hline Liver dysfunction & 3 & 294 & 869 & $1.18(0.55-2.54)$ & 0.68 & $0 \%$ & 0.66 \\
\hline Renal impairment & 1 & 189 & 689 & $0.87(0.33-2.27)$ & 0.77 & NA & NA \\
\hline Radiodermatitis & 5 & 163 & 304 & $1.04(0.81-1.35)$ & 0.74 & $0 \%$ & 0.68 \\
\hline Skin rash & 4 & 358 & 943 & 4.08 (1.59-10.47) & 0.003 & $70 \%$ & 0.02 \\
\hline Mucositis & 7 & 565 & 1152 & $1.84(1.10-3.10)$ & 0.02 & $89 \%$ & $<0.001$ \\
\hline Weight loss & 2 & 264 & 839 & 1.51 (0.95- 2.39) & 0.08 & $0 \%$ & 0.62 \\
\hline \multicolumn{8}{|c|}{ Comparison 2: RT + Concurrent Anti-EGFR mAbs Versus CCRT } \\
\hline Leukopenia & 3 & 251 & 680 & $0.23(0.10-0.53)$ & $<0.001$ & $51 \%$ & 0.13 \\
\hline Thrombocytopenia & 4 & 279 & 712 & $0.27(0.11-0.62)$ & 0.002 & $0 \%$ & 0.59 \\
\hline Anemia & 3 & 227 & 660 & $0.09(0.03-0.33)$ & $<0.001$ & $0 \%$ & 0.92 \\
\hline Nausea/vomiting & 4 & 279 & 712 & $0.10(0.05-0.18)$ & $<0.001$ & $10 \%$ & 0.34 \\
\hline Liver dysfunction & 3 & 227 & 660 & $1.41(0.10-20.76)$ & 0.80 & $60 \%$ & 0.08 \\
\hline Renal impairment & 3 & 227 & 660 & $0.20(0.05-0.79)$ & 0.02 & $48 \%$ & 0.15 \\
\hline Radiodermatitis & 1 & 52 & 52 & $2.00(0.19-21.38)$ & 0.57 & NA & NA \\
\hline Skin rash & 5 & 300 & 735 & 4.09 (1.21-13.87) & 0.02 & $63 \%$ & 0.03 \\
\hline Mucositis & 5 & 300 & 735 & $1.28(0.97-1.68)$ & 0.08 & $61 \%$ & 0.04 \\
\hline Weight loss & 3 & 251 & 680 & $0.96(0.64-1.45)$ & 0.84 & $12 \%$ & 0.32 \\
\hline
\end{tabular}

Abbreviations: $\mathrm{N}=$ number; $\mathrm{pts}=$ patients; anti-EGFR $\mathrm{mAb}=$ anti-epidermal growth factor receptor monoclonal antibody; $\mathrm{RT}=$ radiotherapy; $\mathrm{CRT}=\mathrm{chemoradiotherapy}$; $\mathrm{CCRT}=$ concurrent chemoradiotherapy; $\mathrm{RR}=$ risk ratio; $\mathrm{CI}=$ confidence interval; $\mathrm{NA}=$ not applicable. a Statistically significant results are shown in bold .

The relative efficacy of cetuximab and nimotuzumab in the treatment of NPC is still undetermined. Although several studies have demonstrated cetuximab and nimotuzumab are efficacious in NPC [16, 17, 19, 20], further comparisons between the two mAbs are needed. Considering the current evidence, cetuximab and nimotuzumab both represent good choices for combination with standard therapy in stage III and IV NPC with respect to efficacy, but nimotuzumab would be a better choice due to its more favorable toxicity profile.

\section{Comparison 2: anti-EGFR mAbs failed to be a recommended choice to replace conventional cytotoxic regimes concurrently with $R T$ in the treatment of locoregionally advanced NPC}

When anti-EGFR mAbs were prescribed to replace conventional cytotoxic regimes concurrently with RT, we did not observe significant differences in OS, DFS, LRFS or DMFS. Of the 368 participants in the $\mathrm{mAb}$ group, 241 patients received induction chemotherapy (IC) before RT. IC has been proven to improve DFS, mainly by benefiting DMFS in patients with advanced NPC $[49,50]$. So, we cannot exclude the possibility that benefits from IC may conceal differences between the two modalities. Subgroup analyses revealed nimotuzumab was significantly inferior to conventional cytotoxic regimes concurrent with RT in OS and DFS, while no significant differences were observed in the cetuximab subgroup. This may be due to the intrinsic pharmacological and immunological differences between the two mAbs in this clinical scenario (i.e. concurrent with RT alone) [51, 52]. More evidence is needed to support this suggestion, considering that only two studies with a small sample size were included in each subgroup analysis. With respect to adverse events, hematological, gastrointestinal and renal toxicities, such platinum-related toxicities were less likely in the RT plus mAb group, though mAb-related toxicities such as skin rashes were frequent in this group.

In the light of the current evidence, we do not recommend the replacement of platinum-based chemotherapy with anti-EGFR mAbs during RT in patients with locoregionally advanced NPC. We could not confirm the non-inferiority of RT plus anti-EGFR mAbs compared to CCRT, especially when we took the results of NCT01614938, the closed ahead-ofschedule phase II trial [38], and the subgroup analyses into consideration. However, for patients with stage II $\mathrm{NPC}$, for whom the choice of RT or CRT is still uncertain, treatment with RT plus anti-EGFR mAbs may provide satisfactory efficacy with tolerable toxicities [40]. Additionally, replacement of chemotherapy with anti-EGFR mAbs could be considered for elderly patients or patients with comorbidities who cannot tolerate the toxicities of platinum-based chemotherapy [40]. The ongoing phase III clinical trial NCT02012062 should provide solid evidence in this respect.

\section{Prospects}

Previous studies demonstrated that inhibition of 
EGFR signaling could modulate cellular sensitivity to radiation and enhance tumor cell response to radiation and established a foundation for the clinical investigation of the combination of radiation and anti-EGFR mAbs [53]. We also noticed that most of current studies incorporated the anti-EGFR mAbs concurrently with RT in the curative setting for non-metastatic NPC. However, what are the effects of anti-EGFR mAbs when incorporated into the induction or adjuvant phase? Peng et al. [54] conducted a retrospective analysis suggesting that anti-EGFR $\mathrm{mAbs}$ in combination with IC may be a more effective and promising strategy for patients with locoregionally advanced NPC. Further investigations are required to explore the application of anti-EGFR mAbs in NPC. The phase III RTOG 0522 trial [55] evaluated the potential benefit of adding cetuximab to CCRT in head and neck squamous cell cancer and demonstrated no improvement in PFS or OS. However, a post hoc analysis of RTOG 0522 study [56] found that patients with the KRAS-variant may significantly benefit from the addition of cetuximab to CCRT. In the future, identifying reliable biomarkers that could predict the effects of anti-EGFR mAbs in NPC and optimizing the use of anti-EGFR mAbs will be a promising direction.

\section{Limitations}

Several limitations of these meta-analyses need to be taken into account. Firstly, thirteen of the seventeen studies included were observational, which may introduce confounding factors into the final results. Secondly, we extracted all information from published data other than individual patient data, which may have resulted in publication and reporting bias. Thirdly, the studies adopted different criteria for evaluation of adverse events, which may cause bias to some extent.

\section{Conclusion}

Our meta-analyses showed that adding antiEGFR mAbs to standard therapy can enhance OS, DFS and LRFS in NPC compared to standard therapy alone with tolerable toxicities. However, replacement of conventional platinum-based chemotherapy with anti-EGFR mAbs concurrently with RT is not supported by our analyses. In view of the limitations of our meta-analyses, further phase III RCTs are necessary to provide more solid evidences.

\section{Abbreviations}

CCRT: concurrent chemoradiotherapy; CI: confidence interval; CRT: chemoradiotherapy; DFS: disease-free survival; DMFS: distant metastasis-free survival; EGFR: epidermal growth factor receptor;
HR: hazard ratio; IC: induction chemotherapy; LRFS: locoregional recurrence-free survival; mAbs: monoclonal antibodies; NPC: nasopharyngeal carcinoma; OS: overall survival; RCT: randomized controlled trial; RR: relative risk; RT: radiotherapy.

\section{Supplementary Material}

Supplementary tables.

http://www.jcancer.org/v09p4510s1.pdf

\section{Acknowledgements}

This study was supported by grants from Natural Science Foundation of Guang Dong Province (No. 2017A030312003), Health \& Medical Collaborative Innovation Project of Guangzhou City, China (201803040003), Innovation Team Development Plan of the Ministry of Education (No. IRT_17R110) and Overseas Expertise Introduction Project for Discipline Innovation (111 Project, B14035).

\section{Competing Interests}

The authors have declared that no competing interest exists.

\section{References}

1. Wei KR, Zheng RS, Zhang SW, Liang ZH, Li ZM, Chen WQ. Nasopharyngeal carcinoma incidence and mortality in China, 2013. Chin J Cancer. 2017; 36: 90. doi: 10.1186/s40880-017-0257-9.

2. Lee AW, Sze WM, Au JS, Leung SF, Leung TW, Chua DT, et al. Treatment results for nasopharyngeal carcinoma in the modern era: the Hong Kong experience. Int J Radiat Oncol Biol Phys. 2005; 61: 1107-16. doi: 10.016/j.ijrobp.2004.07.702.

3. Mao YP, Xie FY, Liu LZ, Sun Y, Li L, Tang LL, et al. Re-evaluation of 6th edition of AJCC staging system for nasopharyngeal carcinoma and proposed improvement based on magnetic resonance imaging. Int J Radiat Oncol Biol Phys. 2009; 73: 1326-34. doi: 10.016/j.ijrobp.2008.07.062. Epub 9 Jan 17.

4. Baujat B, Audry H, Bourhis I, Chan AT, Onat H, Chua DT, et al. Chemotherapy in locally advanced nasopharyngeal carcinoma: an individual patient data meta-analysis of eight randomized trials and 1753 patients. Int J Radiat Oncol Biol Phys. 2006; 64: 47-56. doi: 10.1016/j.ijrobp.2005.06.037.

5. Chan AT, Gregoire V, Lefebvre JL, Licitra L, Hui EP, Leung SF, et al. Nasopharyngeal cancer: EHNS-ESMO-ESTRO Clinical Practice Guidelines for diagnosis, treatment and follow-up. Ann Oncol. 2012; 23: vii83-5. doi: 10.1093 /annonc/mds266.

6. Tang LL, Chen YP, Mao YP, Wang ZX, Guo R, Chen L, et al. Validation of the 8th Edition of the UICC/AJCC Staging System for Nasopharyngeal Carcinoma From Endemic Areas in the Intensity-Modulated Radiotherapy Era. J Natl Compr Canc Netw. 2017; 15: 913-9. doi: 10.6004/jnccn.2017.0121.

7. Ma BB, Hui EP, Chan AT. Systemic approach to improving treatment outcome in nasopharyngeal carcinoma: current and future directions. Cancer Sci. 2008; 99: 1311-8. doi: 10.111/j.49-7006.2008.00836.x. Epub 2008 May 21.

8. Winkler GC, Barle EL, Galati G, Kluwe WM. Functional differentiation of cytotoxic cancer drugs and targeted cancer therapeutics. Regul Toxicol Pharmacol. 2014; 70: 46-53. doi: 10.1016/j.yrtph.2014.06.012. Epub Jun 20.

9. Ma BB, Poon TC, To KF, Zee B, Mo FK, Chan CM, et al. Prognostic significance of tumor angiogenesis, Ki 67, p53 oncoprotein, epidermal growth factor receptor and HER2 receptor protein expression in undifferentiated nasopharyngeal carcinoma--a prospective study. Head Neck. 2003; 25: 864-72. doi: 10.1002/hed.10307.

10. Chua DT, Nicholls JM, Sham JS, Au GK. Prognostic value of epidermal growth factor receptor expression in patients with advanced stage nasopharyngeal carcinoma treated with induction chemotherapy and radiotherapy. Int $\mathrm{J}$ Radiat Oncol Biol Phys. 2004; 59: 11-20. doi: 10.1016/j.ijrobp.2003.10.038.

11. Sun W, Long G, Wang J, Mei Q, Liu D, Hu G. Prognostic role of epidermal growth factor receptor in nasopharyngeal carcinoma: a meta-analysis. Head Neck. 2014; 36: 1508-16. doi: 10.002/hed.23481. Epub 2013 Dec 18.

12. Yarden Y, Pines G. The ERBB network: at last, cancer therapy meets systems biology. Nat Rev Cancer. 2012; 12: 553-63. doi: 10.1038/nrc3309.

13. De Luca A, Carotenuto A, Rachiglio A, Gallo M, Maiello MR, Aldinucci D, et al. The role of the EGFR signaling in tumor microenvironment. J Cell Physiol. 2008; 214: 559-67. doi: 10.1002/jcp.21260. 
14. Sigismund S, Avanzato D, Lanzetti L. Emerging functions of the EGFR in cancer. Mol Oncol. 2017; 10: 1878-0261.

15. Pang LY, Saunders L, Argyle DJ. Epidermal growth factor receptor activity is elevated in glioma cancer stem cells and is required to maintain chemotherapy and radiation resistance. Oncotarget. 2017; 8: 72494-512. doi: 10.18632/oncotarget.9868. eCollection 2017 Sep 22.

16. Chan AT, Hsu MM, Goh BC, Hui EP, Liu TW, Millward MJ, et al. Multicenter, phase II study of cetuximab in combination with carboplatin in patients with recurrent or metastatic nasopharyngeal carcinoma. J Clin Oncol. 2005; 23: 3568-76. doi: 10.1200/JCO.2005.02.147. Epub Apr 4.

17. Ma BB, Kam MK, Leung SF, Hui EP, King AD, Chan SL, et al. A phase II study of concurrent cetuximab-cisplatin and intensity-modulated radiotherapy in locoregionally advanced nasopharyngeal carcinoma. Ann Oncol. 2012; 23: 1287-92. doi: 10.093/annonc/mdr401. Epub 2011 Sep 23.

18. Zhang X, Du L, Zhao F, Wang Q, Yang S, Ma L. A Phase II Clinical Trial of Concurrent Helical Tomotherapy plus Cetuximab Followed by Adjuvant Chemotherapy with Cisplatin and Docetaxel for Locally Advanced Nasopharyngeal Carcinoma. Int J Biol Sci. 2016; 12: 446-53. doi: 10.7150/ijbs.12937. eCollection 2016

19. Huang JF, Zhang FZ, Zou QZ, Zhou LY, Yang B, Chu JJ, et al. Induction chemotherapy followed by concurrent chemoradiation and nimotuzumab for locoregionally advanced nasopharyngeal carcinoma: preliminary results from a phase II clinical trial. Oncotarget. 2017; 8: 2457-65. doi: 10.18632/oncotarget.3899.

20. Huang XD, Yi JL, Gao L, Xu GZ, Jin J, Yang WZ, et al. [Multi-center phase II clinical trial of humanized anti-epidermal factor receptor monoclonal antibody h-R3 combined with radiotherapy for locoregionally advanced nasopharyngeal carcinoma]. Zhonghua Zhong Liu Za Zhi. 2007; 29: 197-201. doi: 10.3760/j.issn:0253-3766.2007.03.009.

21. Higgins JP, Altman DG, Gotzsche PC, Juni P, Moher D, Oxman AD, et al. The Cochrane Collaboration's tool for assessing risk of bias in randomised trials. BMJ. 2011; 343: d5928.

22. [Internet] Wells G, Shea B, O'Connell D, Peterson j, Welch V, Losos M, et al. The Newcastle-Ottawa Scale (NOS) for Assessing the Quality of Non-Randomized Studies in Meta-Analysis. Accessed 2018 May. http://www.ohri.ca/programs/clinical_epidemiology/oxford.asp.

23. Parmar MK, Torri V, Stewart L. Extracting summary statistics to perform meta-analyses of the published literature for survival endpoints. Stat Med. 1998; 17: 2815-34.

24. Higgins JP, Thompson SG, Deeks JJ, Altman DG. Measuring inconsistency in meta-analyses. BMJ. 2003; 327: 557-60. doi: 10.1136/bmj.327.7414.557.

25. Begg CB, Mazumdar M. Operating characteristics of a rank correlation test for publication bias. Biometrics. 1994; 50: 1088-101.

26. Wu RR, Wu SX, Zhao C, Xie FY, Gao JM, Hu WH, et al. [Phase II clinical trial of h-R3 combined radiotherapy for locoregionally advanced nasopharyngeal carcinoma]. Ai Zheng. 2007; 26: 874-9.

27. Shao JF, Li SP, Lian YN, Mao JX, Mo YM. Clinical study of nimotuzumab plus nedaplatin combined with concurrent radiotherapy for locally advanced nasopharyngeal carcinoma. Mod Med J China. 2014; 16: 23-6.

28. Tang WB, Yang W, Cao Y, Wu CR, Pan XX, Liu ZH, et al. The efficacy of nimotuzumab combined with conformal radiotherapy and concurrent chemotherapy in locally advanced nasopharyngeal carcinoma. Guangdong Medical Journal. 2012; 33: 1658-62. doi: 10.3969/j.issn.1001-9448.2012.11.054.

29. Yin Z, Yi J, Huang X, Luo J, Wang K, Gao L, et al. Clinical effects of IMRT combined with EGFR monoclonal antibody, concurrent chemoradiotherapy, and IMRT alone in nasopharyngeal carcinoma patients: a retrospective case-control study. Chin J Radiat Oncol 2014; 23: 495-9. doi: 10.3760/cma.j.issn.1004-4221.2014.06.010.

30. Li D, Bai Y, Xie K, Ao R. Efficacy and safety of nimotuzumab combined with nedaplatin and three-dimensional conformal concurrent radiotherapy for nasopharyngeal carcinoma and the impact on TGF- $\beta 1$, S100A8 and S100A9. J Med Res. 2015; 44: 136-9. doi: 10.11969/j.issn.1673-548X.2015.11.038.

31. Lou F, Yang P, Zhang R, Xie XX, Yang JR, Jiang XY, et al. Comparative study of nimotuzumab with radio-chemotherapy and radio-chemotherapy alone in the treatment of locally advanced nasopharyngeal carcinoma. J Clin Res. 2016; 33: 933-5

32. Wang XX. Clinical assessment of the radiotherapy and chemotherapy combined with cetuximabin in the treatment of nasopharyngeal carcinoma. Journal of Clinical Otorhinolaryngology Head and Neck Surgery. 2016; 30: 1229-31. doi: 10.13201/j.issn.1001-1781.2016.15.013.

33. Zeng T, Zhang YL, Li JJ. Analysis of the efficacy of cetuximab combined with chemotherapy and radiotherapy in the treatment of advanced nasopharyngeal carcinoma. Chinese Journal of the Frontiers of Medical Science (Electronic Version). 2016; 8: 45-8. doi: 10.3969/j.issn.1674-7372.2016.04.012.

34. Xia WX, Liang H, Lv X, Wang L, Qian CN, Ye YF, et al. Combining cetuximab with chemoradiotherapy in patients with locally advanced nasopharyngeal carcinoma: A propensity score analysis. Oral Oncol. 2017; 67:167-174.: 10.1016/j.oraloncology.2017.02.026. Epub Mar 2.

35. You $R$, Hua $Y J$, Liu $Y \mathrm{P}$, Yang $\mathrm{Q}$, Zhang $\mathrm{YN}$, Li JB, et al. Concurrent Chemoradiotherapy with or without Anti-EGFR-Targeted Treatment for Stage II-IVb Nasopharyngeal Carcinoma: Retrospective Analysis with a Large Cohort and Long Follow-up. Theranostics. 2017; 7: 2314-24. doi: 10.7150/thno.19710. eCollection 2017.

36. Wang F, Sun Q, Jiang C, Liu T, Rihito A, Masoto S, et al. Additional induction chemotherapy to concurrent chemotherapy and intensity-modulated radiotherapy with or without nimotuzumab in first-line treatment for locoregionally advanced nasopharyngeal carcinoma: a propensity score matched analysis. J Cancer. 2018; 9: 594-603. doi: 10.7150/jca.20461. eCollection 2018

37. Wu LR, Zhu HF, Xu J, Jiang XS, Yin L, Jiang N, et al. Effectiveness of Cetuximab in Combination with Concurrent Chemoradiotherapy in Locoregionally Advanced Nasopharyngeal Carcinoma: A 1:2 Propensity Score-matched Analysis. J Cancer. 2018; 9: 1642-51. doi: 10.7150/jca.23994. eCollection 2018.

38. Xu T, Liu Y, Dou S, Li F, Guan X, Zhu G. Weekly cetuximab concurrent with IMRT aggravated radiation-induced oral mucositis in locally advanced nasopharyngeal carcinoma: Results of a randomized phase II study. Oral Oncol. 2015; 51: 875-9. doi: 10.1016/j.oraloncology.2015.06.008. Epub Jul 7.

39. Liao XY, Kong L, Zheng H, Dai MM, Wang LC, Lu JD, et al. Comparison of efficacy and adverse reactions between radiotherapy combined with cisplatin and radiotherapy combined with nimotuzumab in treatment of locally advanced nasopharyngeal carcinoma. Chin J Radiat Oncol. 2016; 25: 1277-80.

40. Li HM, Li P, Qian YJ, Wu X, Xie L, Wang F, et al. A retrospective paired study: efficacy and toxicity of nimotuzumab versus cisplatin concurrent with radiotherapy in nasopharyngeal carcinoma. BMC Cancer. 2016; 16: 946. doi: 10.1186/s12885-016-2974-x.

41. Wu X, Huang J, Liu L, Li H, Li P, Zhang J, et al. Cetuximab concurrent with IMRT versus cisplatin concurrent with IMRT in locally advanced nasopharyngeal carcinoma: A retrospective matched case-control study. Medicine (Baltimore). 2016; 95: e4926. doi: 10.1097/MD.0000000000004926.

42. You R, Sun R, Hua YJ, Li CF, Li JB, Zou X, et al. Cetuximab or nimotuzumab plus intensity-modulated radiotherapy versus cisplatin plus intensity-modulated radiotherapy for stage II-IVb nasopharyngeal carcinoma. Int J Cancer. 2017; 141: 1265-76. doi: 10.002/ijc.30819. Epub 2017 Jun 23.

43. Gu J, Yin L, Wu J, Zhang N, Huang T, Ding K, et al. Cetuximab and Cisplatin Show Different Combination Effect in Nasopharyngeal Carcinoma Cells Lines via Inactivation of EGFR/AKT Signaling Pathway. Biochem Res Int. 2016; 2016:7016907.: 10.1155/2016/7016907. Epub 2016 May 24.

44. Liu W, Kang M, Qin Y, Wei Z, Wang R. Apoptosis-inducing effects of cetuximab combined with radiotherapy and hypothermia on human nasopharyngeal carcinoma CNE cells. Int J Clin Exp Med. 2015; 8: 2182-9. eCollection 015 .

45. $\mathrm{Xu} C$, Zhang $\mathrm{LH}$, Chen $\mathrm{YP}$ Liu $\mathrm{X}$, Zhou GQ Lin $\mathrm{AH}$, et al. Chemoradiotherapy Versus Radiotherapy Alone in Stage II Nasopharyngeal Carcinoma: A Systemic Review and Meta-analysis of 2138 Patients. J Cancer. 2017; 8: 287-97. doi: 10.7150/jca.17317. eCollection 2017.

46. Yano S, Kondo K, Yamaguchi M, Richmond G, Hutchison M, Wakeling A, et al. Distribution and function of EGFR in human tissue and the effect of EGFR tyrosine kinase inhibition. Anticancer Res. 2003; 23: 3639-50.

47. Thomas M. Cetuximab: adverse event profile and recommendations for toxicity management. Clin J Oncol Nurs. 2005; 9: 332-8. doi: 10.1188/05.CJON.332-338.

48. Ramakrishnan MS, Eswaraiah A, Crombet T, Piedra P, Saurez G, Iyer H, et al. Nimotuzumab, a promising therapeutic monoclonal for treatment of tumors of epithelial origin. MAbs. 2009; 1: 41-8.

49. Sun Y, Li WF, Chen NY, Zhang N, Hu GQ, Xie FY, et al. Induction chemotherapy plus concurrent chemoradiotherapy versus concurrent chemoradiotherapy alone in locoregionally advanced nasopharyngeal carcinoma: a phase 3, multicentre, randomised controlled trial. Lancet Oncol. 2016; 17: 1509-20. doi: 10.016/S470-2045(16)30410-7. Epub 2016 Sep 27.

50. Ribassin-Majed L, Marguet S, Lee AWM, Ng WT, Ma J, Chan ATC, et al. What Is the Best Treatment of Locally Advanced Nasopharyngeal Carcinoma? An Individual Patient Data Network Meta-Analysis. J Clin Oncol. 2017; 35: 498-505. doi: 10.1200/JCO.2016.67.4119. Epub 2016 Dec 5.

51. Mazorra Z, Lavastida A, Concha-Benavente F, Valdes A, Srivastava RM, Garcia-Bates TM, et al. Nimotuzumab Induces NK Cell Activation, Cytotoxicity, Dendritic Cell Maturation and Expansion of EGFR-Specific T Cells in Head and Neck Cancer Patients. Front Pharmacol. 2017; 8:382.: 10.3389/fphar.2017.00382. eCollection 2017.

52. Jie HB, Srivastava RM, Argiris A, Bauman JE, Kane LP, Ferris RL. Increased PD-1(+) and TIM-3(+) TILs during Cetuximab Therapy Inversely Correlate with Response in Head and Neck Cancer Patients. Cancer Immunol Res. 2017; 5: 408-16. doi: 10.1158/2326-6066.CIR-16-0333. Epub 2017 Apr 13.

53. Morris ZS, Harari PM. Interaction of radiation therapy with molecular targeted agents. J Clin Oncol. 2014; 32: 2886-93.

54. Peng H, Tang LL, Liu X, Chen L, Li WF, Mao YP, et al. Anti-epidermal growth factor receptor therapy concurrently with induction chemotherapy in locoregionally advanced nasopharyngeal carcinoma. Cancer Sci. 2018; 25: 13589 .

55. Ang KK, Zhang Q, Rosenthal DI, Nguyen-Tan PF, Sherman EJ, Weber RS, et al. Randomized phase III trial of concurrent accelerated radiation plus cisplatin with or without cetuximab for stage III to IV head and neck carcinoma: RTOG 0522. J Clin Oncol. 2014; 32: 2940-50.

56. Weidhaas JB, Harris J, Schaue D, Chen AM, Chin R, Axelrod R, et al. The KRAS-Variant and Cetuximab Response in Head and Neck Squamous Cell Cancer: A Secondary Analysis of a Randomized Clinical Trial. JAMA Oncol. 2017; 3: 483-91. doi: 10.1001/jamaoncol.2016.5478. 